Volume 10, No.4, July - August 2021

International Journal of Advanced Trends in Computer Science and Engineering

Available Online at http://www.warse.org/IJATCSE/static/pdf/file/ijatcse041042021.pdf

https://doi.org/10.30534/ijatcse/2021/041042021

\title{
Collaborative Decision Making in IoT Network for Sustainable Smart Cities: An Artificial Intelligence Planning Method Based Solution
}

\author{
Shachi Sharma ${ }^{1}$ \\ ${ }^{1}$ Department of Computer Science, South Asian University, New Delhi, India, shachi@ sau.int
}

\begin{abstract}
The role of artificial intelligence (AI) along with Internet of Things (IoT) has been widely recognized in building intelligent solutions for sustainable smart cities. However, the focus of ongoing research is limited to utilizing machine learning paradigm. Artificial intelligence is a vast field consisting of many diverse methods and techniques. The paper presents a system based on AI planning method that can generate intelligent collaborative decisions for IoT devices by processing the real-time data from IoT devices. A usecase of adaptive traffic signal explains the working of the system and also proves its feasibility.
\end{abstract}

Key words : Artificial intelligence, collaborative decisions, IoT, planning method, smart cities

\section{INTRODUCTION}

Recent years have drawn considerable attention on growing urbanization of the world population. According to a United Nation report [1], two-third of the world population will be living in the cities by year 2050 . There are multiple reasons for this urbanization ranging from better job and education opportunities to health infrastructure available in cities. The huge growth of urban population poses multiple problems such as traffic congestion, pollution, crime etc. which are also threats to sustainable cities. One solution lies in adopting the science and technology advancements to address these challenges converting cities into smart sustainable cities. According to ITU-T [2], "A smart sustainable city (SSC) is an innovative city that uses information and communication technologies (ICT) and other means to improve: quality of life, efficiency of urban operation and services, and competitiveness; while ensuring that it meets the needs of present and future generations, with respect to: economic, social, environmental, and cultural aspects". Another definition of smart city is given jointly by ISO and IEC [3] as "Smart Cities provide a new concept and a new model, which applies the new generation of information technologies, such as the internet of things, cloud computing, big data and space geographical information integration, to facilitate the planning, construction, management and smart services of cities. Developing Smart Cities can benefit synchronized development, industrialization, informationization, urbanization and agricultural modernization and sustainability of cities development". While the focus of ITU-T is on improving the quality of life using ICT, the ISO and IEC pin-point to the specific technologies for providing smart services.

Two promising technologies that play major role in developing solutions for converting cities into smart cities are IoT and AI [4]. The prime objective of IoT is to identify and connect all the things around to the internet [5]. Radio frequency identifiers (RFID), sensors, actuators and smart phones are considered to be key enablers for IoT. The terms 'objects', 'things' and 'devices' are used interchangeably in IoT domain. It is expected that the growth of connected IoT devices will reach 1.25 billion by year 2030 [6] and these devices will produce huge amount of data [7]. This data can be analyzed to draw useful and meaningful inferences using AI techniques enabling devices to learn and behave smartly, also make decisions autonomously. The integration of $\mathrm{AI}$ and IoT is revolutionary and leading to realization of many complex solutions such as driverless cars [8].

Recognizing the potential of $\mathrm{AI}$ and IoT in converting cities into smart cities, several studies have been carried out in the past. A detailed survey of available IoT technologies such as protocols and devices for smart cities is provided in [9]. A potential IoT network architecture based on web-services with its proof-of-concept deployment in Padova city of Italy is presented in [10]. A new method, called AAIoT, is proposed in [10] that overloads deep learning computational tasks in multi-layer IoT system intelligently to reduce response time. A performance study of smart cities specific IoT communication systems is carried out using deep learning in [11]. An intelligent accident reducing system called Smart Accident Precognition System (SAPS) is proposed in which various embedded devices in the vehicle monitor speed, distance, safety measures etc. and send the sensed data to 
cloud for analysis using machine learning [12]. Iddianozie and Palmes [13] propose a Building Management System (BMS) that decreases energy consumption of buildings. The BMS system consists of smart devices and machine learning algorithms based on Image Encoded Time Series (IETS) analysis to deduce from multi-dimensional data. A review of IoT based smart energy system is provided in [14]. A smart parking system for smart cities is presented in [15]. Chithaluru et al. develop a new protocol for green IoT in smart cities [16]. An AI driven IoT based ehealth architecture is proposed by Patam et al. [17]. It deploys a new grey filter Bayesian convolution neural network for analysis of real-time data coming from wearables of patients. Providing robust security and privacy is also essential for smart cities. A smart intrusion detection system using distributed parallel machine models is discussed in [18]. A smart bin solution is proposed in [19].

To overcome the limitations of traditional centralized machine learning model of AI, a new federated machine learning paradigm has gained prominence in last few years. Federated machine learning allows to train a model without sharing private and secure data [20]. A detailed discussion on applications of federated machine learning in IoT leading to FL-IoT intelligent services such as smart healthcare, unmanned aerial vehicles and other smart city solutions have been provided in [20]. The prime focus in most of the present studies is on using machine learning methods for analysis of data generated by IoT devices. However, AI is a vast and mature field with many methods for handling data. The feasibility of those AI techniques should also be explored to build efficient intelligent systems for sustainable smart cities. This is the main motivation behind this work.

The paper presents an AI planning method based model that collects real-time streaming data from multiple IoT devices such as sensors, aggregates data, and generates decisions in real-time to control devices like actuators automatically. An application of the proposed model in adaptive traffic signals is also discussed. However, the proposed model is generic and can be applied to any problem where collaborative decision making is required in real-time. The paper is organized into five sections. A brief discussion of AI planning method is carried out in section 2. The planning method based system for collaborative and intelligent decision making is presented in section 3. The adaptive traffic signal usecase built using the proposed system is presented in section 4 . The last section 5 contains conclusion.

\section{OVERVIEW OF AI PLANNING METHOD}

The prime objective of AI planning methods is to select and order a set of actions, also called steps, required to fulfill a goal [21]. A typical planning problem is represented by a triplet containing a domain model, an initial state and a goal state. The domain model describes the objects in the problem, the actions that can be applied on these objects, and the constraints on the actions. The actions are defined in the form of operators. The initial state signifies the state before any action are applied and the goal state represents the facts that must be satisfied once the plan has been executed completely.

Usually, a planning task is divided into two related subtasks. The first one is dedicated on finding the required steps to solve the problem. The second subtask resolves the conflicts between the steps generated by the first subtask. The conflicts can be rectified by reordering actions, adding new actions or by complete replanning (i.e., redoing subtask 1) as discussed in [22].

One of the major problems while using planning method is of large state space. Hence, a lot of research work in the domain of planning algorithm deals with this aspect. One popular approach is least-commitment planning [21], a non-linear approach, where a set of plans are represented together by introducing only required constraints between actions. Another notable solution is Hierarchical Task Network (HTN) planning [23] where the problem is decomposed into multiple sub-problems represented as a hierarchy. The higher levels in the hierarchy provide abstraction of the problem and lower levels detail the problem. Hence, at each level only a part of the problem is solved thereby reduces the search space.

The common language to represent a problem and solve it in AI planner is Planning Domain Definition Language (PDDL) and the same has been used in this research work. A good literature on PDDL is presented in [24] recently.

\section{THE PROPOSED SYSTEM}

The key to the IoT network is sensors that produce huge volume of data that needs to be processed to draw useful information in almost real-time. This information can be utilized to make automated decisions such as for managing traffic or utilities (electricity, water or gas) in smart cities. The problem can be defined as, "Is it possible to use real-time streaming data generated by IoT devices to generate decisions to control other devices or actuators automatically?"

The AI planner can be used to make decisions from the real-time data. A high-level overview of the system architecture is provided in Figure 1. First, the data is collected from the IoT devices, for example, sensors in vehicles. Then the model of the problem is created and both the collected data and model are fed to the AI planner. The planner generates a set of decisions which are passed back to the devices for taking actions. 
The low-level design of the proposed system along with the flow inside the system is illustrated in Figure 2. Because of the heterogeneity of IoT devices, the data produced by them varies in the format. Hence, the data collection module is subdivided into two parts - one that communicates with devices and gathers data and other that cleans and converts the data in a standard format, also sends it to AI planner. The first module is called data collector and second is data feeder.
The advantages of such a partitioning of functionalities are manifold. One it is a neat design, second, with the addition of new type of devices, only the data collector module will undergo change. This makes design robust. The other module that interacts with IoT devices is data transmitter responsible for sending decisions to devices. This module too needs to support the protocol and other communication technologies supported by devices. Automatic planner combines data with model and generates decisions. The steps are summarized below,

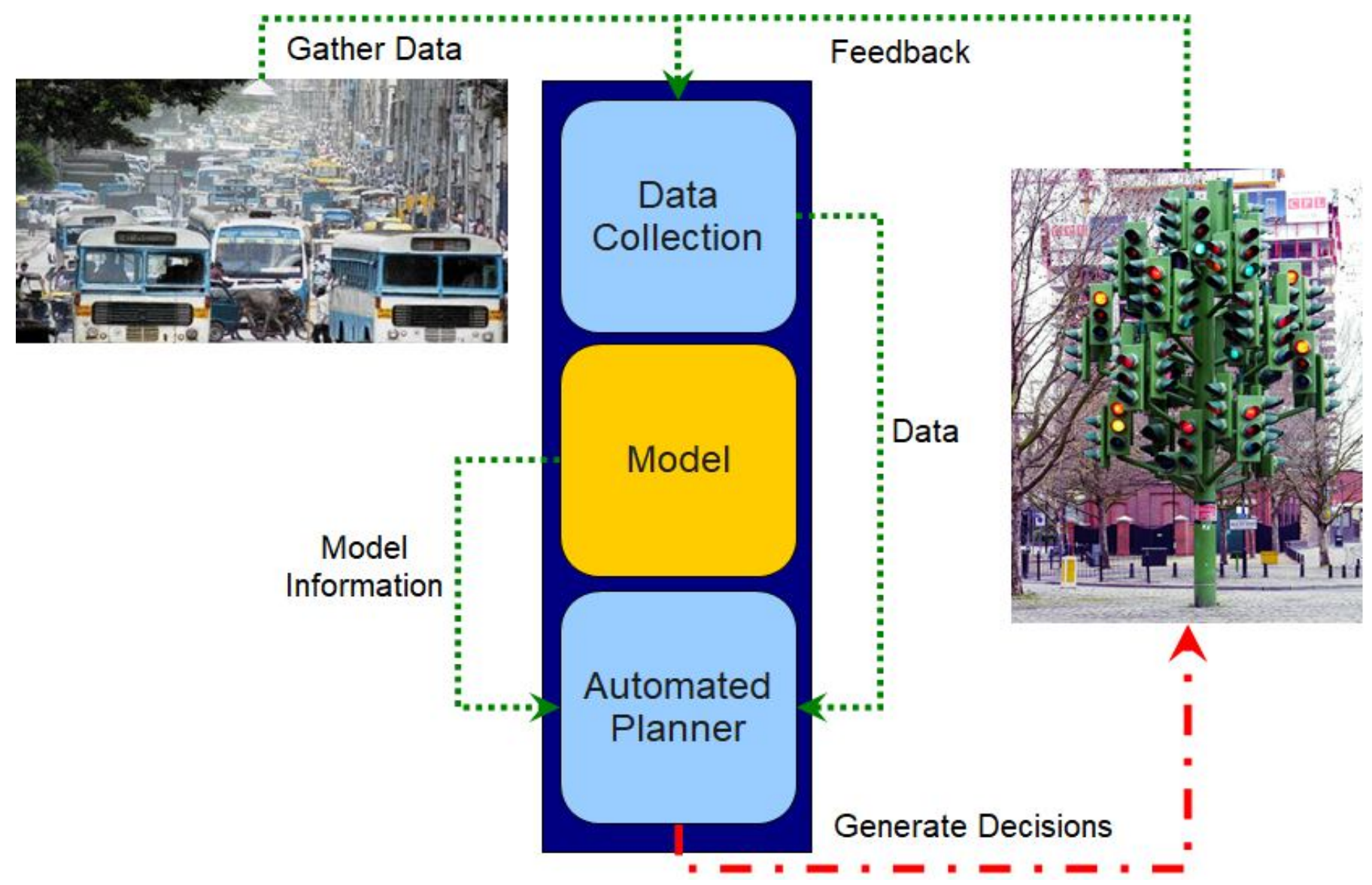

Figure 1: A high-level architecture of the proposed AI Planner-based system. 


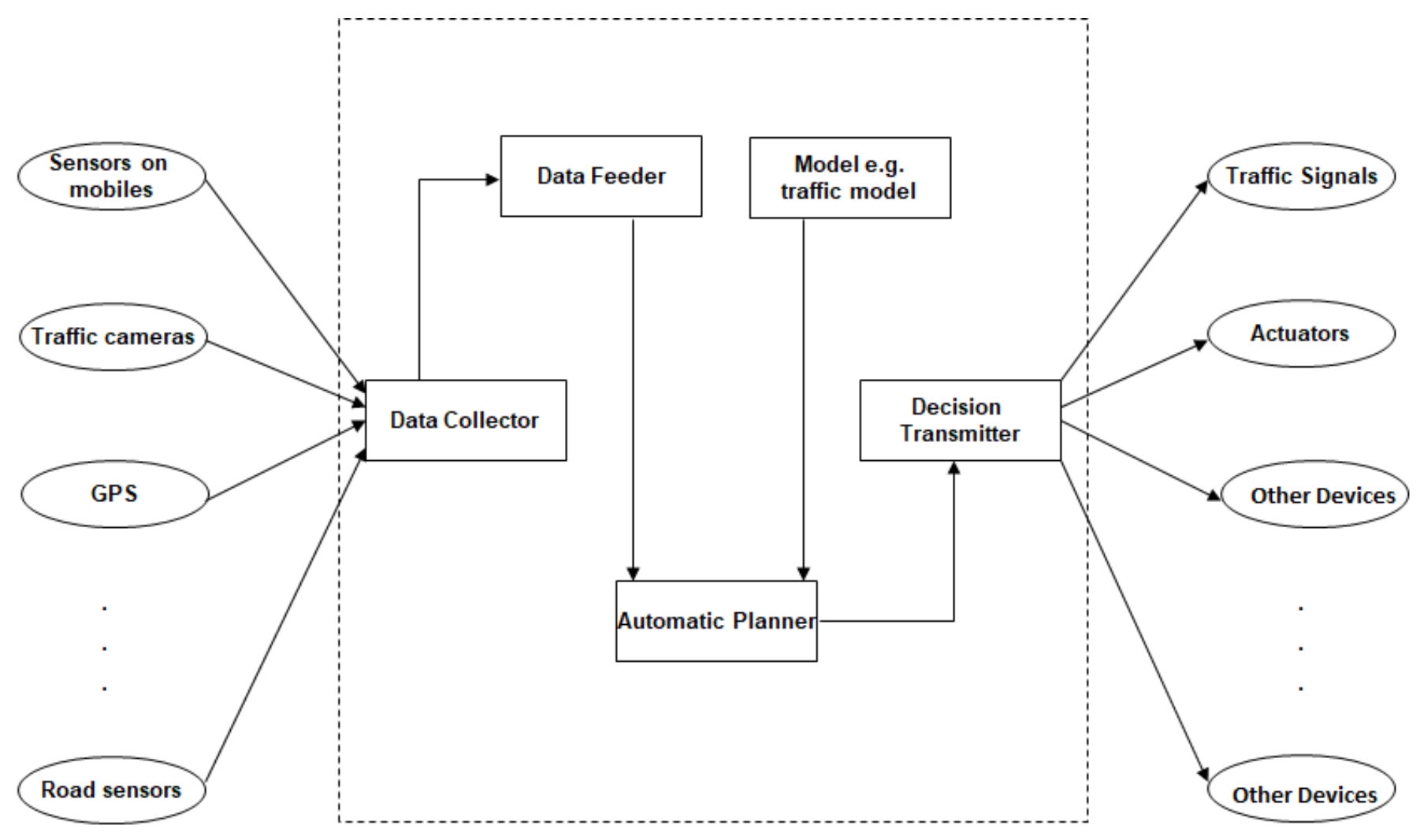

Figure 2: Details of the procedure followed in the system.

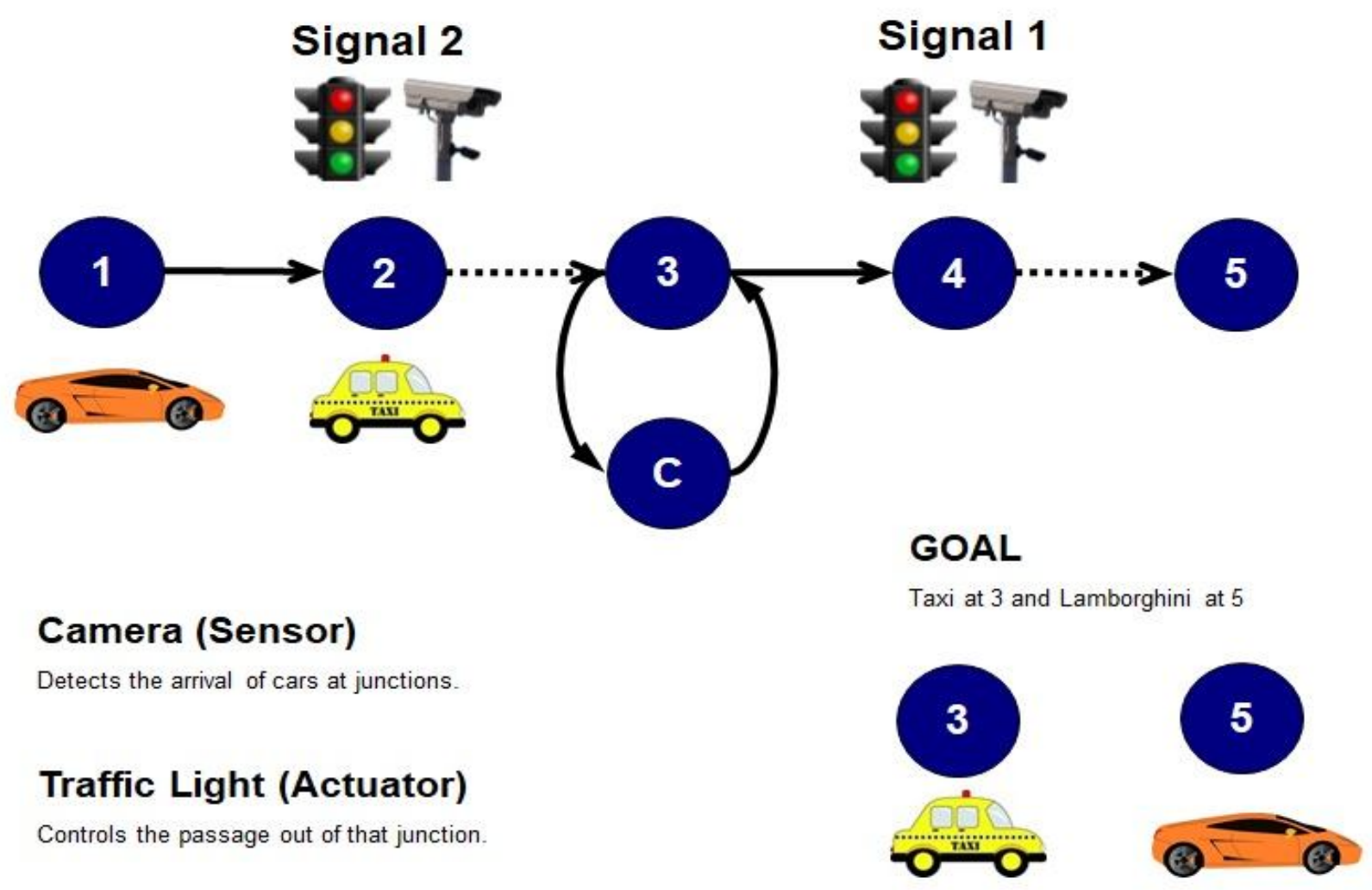

Figure 3: Usecase-Adaptive traffic signal. 
- design the model of the problem

- collect data from sensors and other IoT devices

- clean and convert the data into a standardized format

- feed the data and model to an automated planner to return decisions

- convert the decisions of the planner in required format and transmit them to actuators and devices.

The system design is flexible as any model can be plugged into the system. In order to support new IoT devices, data collector and transmitter modules only are required to be upgraded. Thus, the system design is robust.

The system is used to create adaptive traffic signals in smart cities. The details of this usecase are provided in the next section.

\section{USECASE: ADAPTIVE TRAFFIC SIGNAL}

The AI Planning based system is used to construct adaptive traffic signals as an usecase. The AI modeling language used is PDDL, the standard modeling language in the automated planning community.

In normal cases, the duration of a traffic signal to turn green, yellow or red is either fixed or is controlled manually by the traffic controlling agency like traffic police. This often leads to congestion on roads where number of vehicles is more whereas the signal unnecessary remains green for roads without vehicle. Such inefficiencies are not desirable in a smart city. To overcome this, adaptive traffic signal usecase has been constructed. The duration of the traffic signals to turn green or red is determined by the number of approaching vehicles. IoT devices like the sensors on the road, phone, camera mounted on the traffic signal can report the incoming vehicles at a traffic intersection as data stream. An example is shown in Figure 3 where two cars (a taxi and a Lamborghini) are first approaching the traffic signal 2. The destination of taxi is 3 and that of Lamborghini is 5. Hence, Lamborghini also needs to cross traffic signal 1 .

The various objects for this usecase can be represented in the PDDL as

(:types

locsensor visualsensor roadsensor rfsensor - sensor gpssensor phonesensor longrangerf - locsensor camera laser - visualsensor inductiveloop - roadsensor tollreader - rfsensor truck bus car auto bike - vehicle vehicle pedestrian - carrier segment - location signal gate - actuator sensor carrier actuator - locatable locatable location actuator - object)

There are two parts of the traffic model,

- Predicates: They are Boolean facts about the objects in the scenario. Predicates can be defined in PDDL as

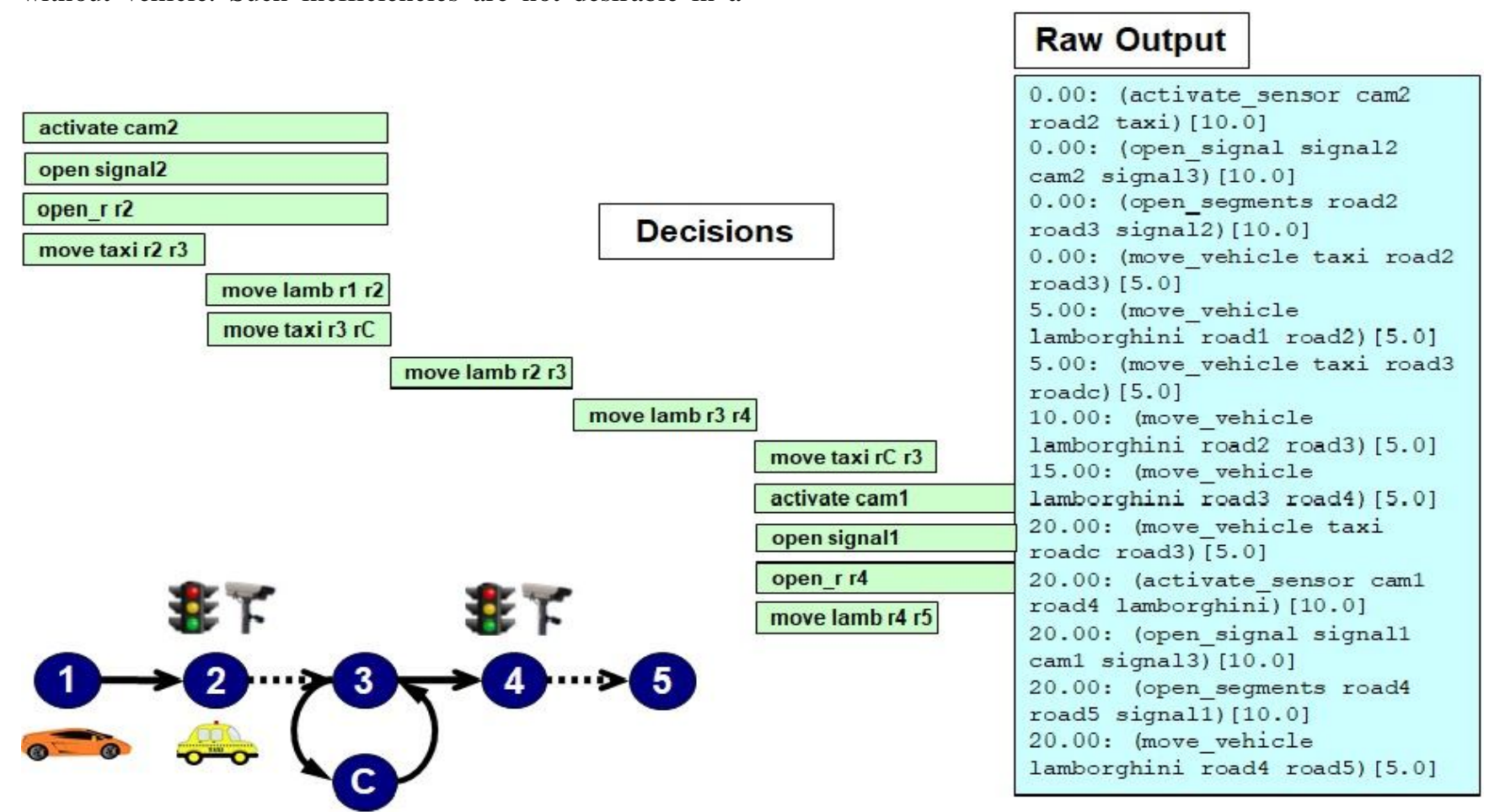

(:predicates

Figure 4: Output of the system for usecase example (at ?obj - locatable ?loc - location) 


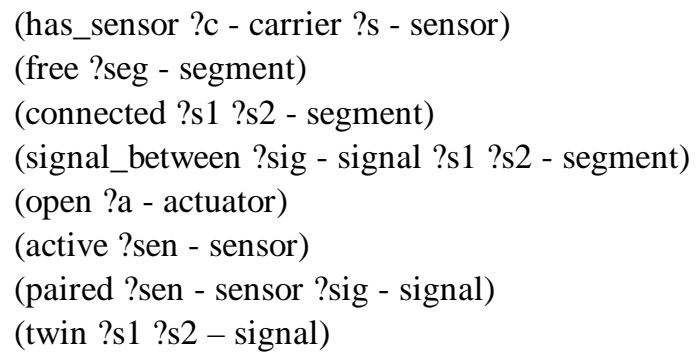

- Decisions: These are the most important aspects of the scenario and involves various sensors and actuators. Some examples of decisions representation in PDDL are

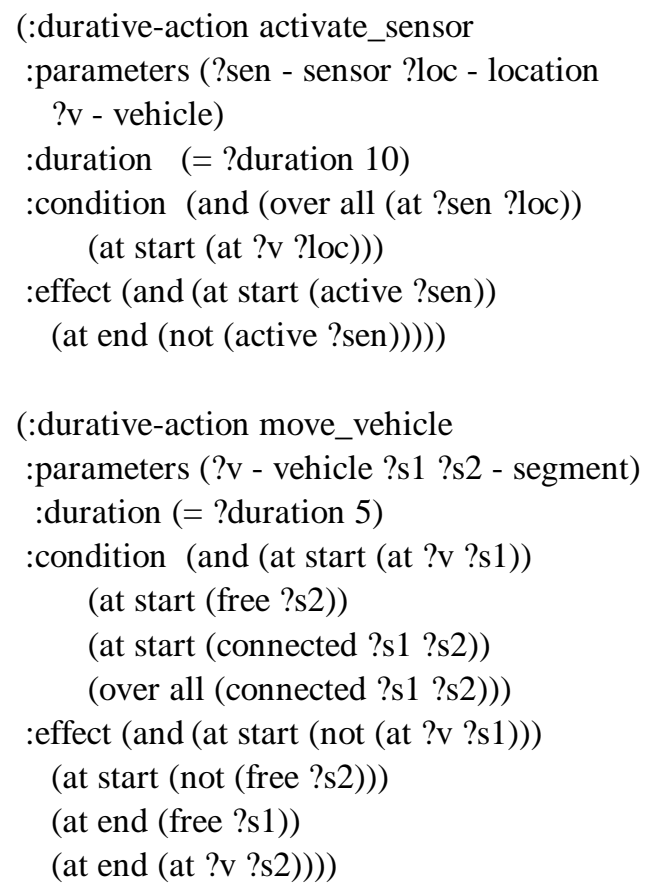

The output by the system for usecase of Figure 3 is shown in Figure 4. As the taxi and Lamborghini come on road 2 and this event is reported by the road sensors, the camera 2 is switched on and thereafter the signal 2 is turned on to green as the vehicles are approaching. As the Lamborghini approaches on road 3 towards its destination 5 , the event is reported by road sensors, camera 1 is turned on and the traffic signal 1 is also turned to green. This small example demonstrates how the AI planning based system can be used to generate decisions to turn on cameras and traffic signals. This saves a lot of electricity and other resources. Such a system can also be used to create a passage for emergency vehicles such as ambulances and fire trucks.

\section{CONCLUSION}

A novel system utilizing AI planning method for generating collaborative intelligent decisions from real-time streaming data from multiple IoT devices has been proposed. The key to the system architecture is model of the problem that can be plugged into the system using PDDL. The feasibility and working of the system are demonstrated by constructing a usecase of adaptive traffic signal for sustainable smart cities. The paper also emphasizes on the requirement of exploring the application of other AI techniques like planning method, besides machine learning, to build intelligent solutions in future.

\section{REFERENCES}

1. United Nations. World urbanization prospects: the 2018 revision. https://populationun.org/wup/

2. ITU (International Telecommunication Union). Technical report \& specifications (ITU-T's): shaping smarter and more sustainable cities: striving for sustainable development, 2016.

3. ISO-IEC (International Standards Organization-International Electrotechnical Commission). Information technology, smart cities: preliminary report, 2014.

4. M. A. Ahad, S. Paiva, G. Tripathi, and N. Feroz. Enabling technologies and sustainable smart cities, Sustainable Cities and Societies, vol. 61, 2020. https://doi.org/10.1016/j.scs.2020.102301

5. S. Sharma. Attribute based discovery architecture for devices in Internet of Things (IoT), in 5th Intl. Conf. on Convergence in Technology, 2019.

6. V. Marletta. Life after graduation: IoT: Forecasts, challenges and opportunities, IEEE Instrum. \& Measurem. Mag., vol.22, no. 6, pp. 76-77, 2019.

7. A. Al-Fuqaha et al. Internet of things: A survey on enabling technologies, protocols, and applications, IEEE Commun. Surveys and Tutorials, vol. 17, pp. 2347-2376, 2015.

8. Z. Allama, and Z. A. Dhunny. On big data, artificial intelligence and smart cities, Cities, vol. 89, pp. 80-91, 2019.

9. A. Zanella, et al. Internet of things for smart cities, IEEE Internet of Things J., vol. 1, no.1, pp. 22-32, 2014.

10. J. Zhou et al. AAIoT: Accelerating artificial intelligence in IoT systems, IEEE Wireless Commun. Letts., vol. 8, no. 3, pp. 825-828, 2019.

11. A. N. Muhammad, et al. Deep learning application in smart cities: recent development, taxonomy, challenges and research prospects, Neural Computing and Applications, vol. 33, pp. 2973-3009, 2021.

12. V. G. Menon, et al. An IoT-enabled intelligent automobile system for smart cities, Internet of Things, 2020. https://doi.org/10.1016/j.iot.2020.100213

13. C. Iddianozie, and P. Palmes. Towards smart sustainable cities: Addressing semantic heterogeneity in Building Management Systems using discriminative models, Sustainable Cities and Society, vol. 62, 2020. 
14. T. Ahmad, and D. Zhang. Using the internet of things in smart energy systems and networks, Sustainable Cities and Society, vol. 68, 2021.

15. F. Al-Turjman, and A. Malekloo. Smart parking in IoT-enabled cities: A survey, Sustainable Cities and Society, vol. 49. 2019.

16. P. Chithaluru, et al. I-AREOR: An energy-balanced clustering protocol for implementing green IoT in smart cities, Sustainable Cities and Society, vol. 61, 2020.

17. R. Patan, et al. Smart healthcare and quality of service in IoT using grey filter convolutional based cyber physical system, Sustainable Cities and Society, vol. 59, 2020.

18. Md A. Rahman, et al. Scalable machine learning-based intrusion detection system for IoT-enabled smart cities, Sustainable Cities and Society, vol. 61, 2020.

19. C. A. Correya, et al. Smart electronic bin using IoT, Intl. J. of Advanced Trends in Computer Sci. and Engineering, vol. 10, no. 3, pp. 2178-2182, 2021.

20. D. C. Nguyen, et al. Federated learning for internet of things: a comprehensive survey, IEEE Commun. Surveys \& Tutorials, 2021. 10.1109/COMST.2021.3075439

21. D. S. Weld. An Introduction to Least Commitment Planning. AI Mag., vol. 15, no. 4, pp. 27-61, 1994. https://doi.org/10.1609/aimag.v15i4.1109

22. R. S. Aylett, G. J. Petley, P. W. H. Chung, B. Chen, and D. W Edwards. AI planning: solutions for real world problems, Knowledge-Based Systs., vol. 13, no. 2-3, pp. 61-69, 2000.

23. I. Georgievski and M. Aiello. HTN planning: Overview, comparison, and beyond, Artifi. Intelli,, vol. 222, pp. 124-156.

24. P. Haslum, N. Lipovetzky, D. Magazzeni, and C. Muise. An Introduction to the Planning Domain Definition Language, Morgan \& Claypool, 2019. 